Pacific Journal of Mathematics

RANSCENDENTAL ADDITION THEOREMS FOR THE 


\title{
TRANSCENDENTAL ADDITION THEOREMS FOR THE HYPERGEOMETRIC FUNCTION OF GAUSS
}

\author{
F. M. RAGAB
}

1. Introduction. In this paper, integrals involving products of two Gauss functions, regarded as functions of their parameters, are evaluated it terms of other functions of the same kind. In all these integrals it is assumed that $|x|<1$. Also the integrals are taken up the entire length of the imaginary axis with loops, if necessary, to separate the increasing and decreasing sequences of poles. These formulae are :

$$
\begin{aligned}
& \frac{1}{2 \pi i} \int \frac{\Gamma(\alpha+s) \Gamma\left(\alpha^{\prime}-s\right)}{\Gamma^{\prime}(\gamma+s) \Gamma\left(\gamma^{\prime}-s\right)} F(\alpha+s, \beta ; \gamma+s ; x) F\left(\alpha^{\prime}-s, \beta^{\prime} ; \gamma^{\prime}-s ; x\right) d s \\
& \quad=\frac{\Gamma^{\prime}\left(\alpha+\alpha^{\prime}\right) \Gamma\left(\gamma+\gamma^{\prime}-\alpha-\alpha^{\prime}-1\right)}{\Gamma^{\prime}\left(\gamma+\gamma^{\prime}-1\right) \Gamma(\gamma-\alpha) \Gamma\left(\gamma^{\prime}-\alpha^{\prime}\right)} F\left(\alpha+\alpha^{\prime}, \beta+\beta^{\prime} ; \gamma+\gamma^{\prime}-1 ; x\right),
\end{aligned}
$$

where $\gamma+\gamma^{\prime}-1 \neq 0,-1,-2, \cdots ; \gamma-\alpha \neq 0,-1,-2, \cdots$ and $\gamma^{\prime}-\alpha^{\prime} \neq 0$, $-1,-2, \cdots$;

$$
\begin{aligned}
& \frac{1}{2 \pi i} \int \frac{\Gamma(\alpha+s) \Gamma\left(\alpha^{\prime}-s\right)}{\Gamma(\beta+s) \Gamma\left(\beta^{\prime}-s\right)} F(\beta-\alpha, \gamma ; \beta+s ; x) F\left(\beta^{\prime}-\alpha^{\prime}, \gamma^{\prime} ; \beta^{\prime}-s ; x\right) d s \\
= & \frac{\Gamma\left(\alpha+\alpha^{\prime}\right) \Gamma\left(\beta+\beta^{\prime}-\alpha-\alpha^{\prime}-1\right)}{\Gamma(\beta-\alpha) \Gamma\left(\beta^{\prime}-\alpha^{\prime}\right) \Gamma\left(\beta+\beta^{\prime}-1\right)} F\left(\beta+\beta^{\prime}-\alpha-\alpha^{\prime}-1, \gamma+\gamma^{\prime} ; \beta+\beta^{\prime}-1 ; x\right),
\end{aligned}
$$

where $\beta-\alpha \neq 0,-1,-2, \cdots ; \beta^{\prime}-\alpha^{\prime} \neq 0,-1,-2, \cdots$ and $\beta+\beta^{\prime}-1 \neq 0$, $-1,-2, \cdots$;

$$
\begin{aligned}
\frac{1}{2 \pi i} \int & \Gamma(\alpha+s) \Gamma\left(\alpha^{\prime}+s\right) \Gamma(\beta-s) \Gamma\left(\beta^{\prime}-s\right) F\left(\alpha+s, \beta-s ; \frac{1}{2} \alpha+\frac{1}{2} \beta+\frac{1}{2} ; x\right) \\
& \times F\left(\alpha^{\prime}+s, \beta^{\prime}-s ; \frac{1}{2} \alpha^{\prime}+\frac{1}{2} \beta^{\prime}+\frac{1}{2} ; x\right) d s \\
= & \Gamma\left(\alpha+\beta^{\prime}\right) \Gamma\left(\alpha^{\prime}+\beta\right) \Gamma(\alpha+\beta) \Gamma\left(\alpha^{\prime}+\beta^{\prime}\right)\left\{\Gamma\left(\alpha+\alpha^{\prime}+\beta+\beta^{\prime}\right\}^{-1}\right. \\
& \times F\left(\alpha+\beta^{\prime}, \alpha^{\prime}+\beta ; \frac{1}{2} \alpha+\frac{1}{2} \alpha^{\prime}+\frac{1}{2} \beta+\frac{1}{2} \beta^{\prime}+\frac{1}{2} ; x\right),
\end{aligned}
$$

where $\alpha+\alpha^{\prime}+\beta+\beta^{\prime} \neq 0,-1,-2, \cdots$;

$$
\begin{aligned}
\frac{1}{2 \pi i} \int & \Gamma(\gamma+s) \Gamma\left(\gamma^{\prime}-s\right) \Gamma(\alpha+s) \Gamma\left(\alpha^{\prime}-s\right) \\
& \quad \times F\left(\alpha+s, \beta ; \alpha+\gamma^{\prime} ; x\right) F\left(\alpha^{\prime}-s, \beta^{\prime}, \alpha^{\prime}+\gamma ; x\right) d s \\
= & \Gamma\left(\gamma+\gamma^{\prime}\right) \Gamma\left(\alpha+\alpha^{\prime}\right) \Gamma\left(\alpha+\gamma^{\prime}\right) \Gamma\left(\alpha^{\prime}+\gamma\right)\left\{\Gamma\left(\alpha+\alpha^{\prime}+\gamma+\gamma^{\prime}\right)\right\}^{-1} \\
& \quad \times F\left(\alpha+\alpha^{\prime}, \beta+\beta^{\prime} ; \alpha+\alpha^{\prime}+\gamma+\gamma^{\prime} ; x\right),
\end{aligned}
$$

Received March 22, 1957, and in revised form July 19, 1957. 
where $\alpha+\alpha^{\prime}+\gamma+\gamma^{\prime} \neq 0,-1,-2, \cdots ;$ and

$$
\begin{aligned}
& \frac{1}{2 \pi i} \int \frac{\Gamma(\beta+s) \Gamma\left(\beta^{\prime}-s\right)}{\Gamma^{\prime}(\gamma+s) \Gamma^{\prime}\left(\gamma^{\prime}-s\right)} F(\alpha+s, \beta+s ; \gamma+s ; x) F\left(\alpha^{\prime}-s, \beta^{\prime}-s ; \gamma^{\prime}-s ; x\right) d s \\
& =\frac{\Gamma\left(\gamma+\gamma^{\prime}-\beta-\beta^{\prime}-1\right) \Gamma\left(\beta+\beta^{\prime}\right)}{=} F\left(\beta+\beta^{\prime}, \alpha+\alpha^{\prime}-1 ; \gamma+\gamma^{\prime}-1 ; x\right), \\
& \Gamma(\gamma-\beta) \Gamma\left(\gamma^{\prime}-\beta^{\prime}\right) \Gamma\left(\gamma+\gamma^{\prime}-1\right)
\end{aligned}
$$

where $\gamma-\beta \neq 0,-1,-2, \cdots ; \gamma^{\prime}-\beta^{\prime} \neq 0,-1,-2, \cdots$ and $\gamma+\gamma^{\prime}-1 \neq 0,-1$, $-2, \cdots$.

All we need is the following two formulae [E. C. Titchmarch, Fourier integrals, p. 194] :

$$
\begin{aligned}
& 1 \\
& 2 \pi i \\
& \quad=\Gamma(\alpha+\gamma) \int_{k-i \alpha}^{k+i \infty} \Gamma(\alpha+s) \Gamma^{\prime}(\alpha+\delta) \Gamma(\beta+\gamma) \Gamma(\gamma-s) \Gamma(\delta-s) d s \\
& \quad \Gamma(\beta+\delta)\{\Gamma(\alpha+\beta+\gamma+\delta)\}^{-1}
\end{aligned}
$$

where $(-\alpha<k,-\beta<k, \gamma>k, \delta>k)$; and

$$
\frac{1}{2 \pi i} \int_{k-i \infty}^{k+i \infty} \Gamma(\alpha-s) \Gamma(\gamma-s) d s=\frac{\Gamma(\alpha+\gamma) \Gamma(\beta+\delta-\alpha-\gamma-1)}{\Gamma(\beta-\alpha) \Gamma^{\prime}(\delta-\gamma) \Gamma(\beta+\delta-1)},
$$

where $(-\alpha<k,-\beta<k, \gamma>k, \delta>k)$.

It may be noted that the restrictions, needed for (6) and (7), on the parameters in formulae (1)-(5), can be removed later on by the theory of analytical continuation. The proofs and two other formulae will be given in $\S 2$, , while some confluent forms of addition theorems will be deduced, as a limiting case, in $\S 3$.

2. Proof. On expanding each hypergeometric function on the left hand side of (1) and changing the order of integration and summation it becomes

$$
\sum_{m=0}^{\infty} \sum_{n=0}^{\infty} \frac{(\beta ; m)\left(\beta^{\prime} ; n\right)}{m ! n !} x^{m+n} \cdot \frac{1}{2 \pi i} \int \frac{\Gamma(\alpha+m+s) \Gamma\left(\alpha^{\prime}+n-s\right)}{\Gamma^{\prime}(\gamma+m+s) \Gamma\left(\gamma^{\prime}+n-s\right)} d s .
$$

From (7), it follows that the last integral is equal to

$$
\frac{\Gamma\left(\alpha+\alpha^{\prime}+m+n\right) \Gamma\left(\gamma+\gamma^{\prime}-\alpha-\alpha^{\prime}-1\right)}{\Gamma(\gamma-\alpha) \Gamma\left(\gamma^{\prime}-\alpha^{\prime}\right) \Gamma\left(\gamma+\gamma^{\prime}+m+n-1\right)} .
$$

Thus the left hand side of (1) becomes

$$
\begin{aligned}
& \Gamma\left(\alpha+\alpha^{\prime}\right) \Gamma\left(\gamma+\gamma^{\prime}-\alpha-\alpha^{\prime}-1\right) \\
& \Gamma\left(\gamma^{\prime}-\alpha^{\prime}\right) \Gamma(\gamma-\alpha) \Gamma\left(\gamma+\gamma^{\prime}-1\right) \\
& \quad \times \sum_{m=0}^{\infty} \sum_{n=0}^{\infty} \frac{(\beta ; m)\left(\beta^{\prime} ; n\right)\left(\alpha+\alpha^{\prime} ; m+n\right)}{m ! n !\left(\gamma+\gamma^{\prime}-1 ; m+n\right)}-x^{m+n}
\end{aligned}
$$




$$
\begin{aligned}
= & \frac{\Gamma\left(\alpha+\alpha^{\prime}\right) \Gamma\left(\gamma+\gamma^{\prime}-\alpha-\alpha^{\prime}-1\right)}{I^{\prime}\left(\gamma^{\prime}-\alpha^{\prime}\right) \Gamma(\gamma-\alpha) \Gamma\left(\gamma+\gamma^{\prime}-1\right)} \\
& \times \sum_{p=0}^{\infty} \frac{\left(\alpha+\alpha^{\prime} ; p\right)(\beta ; p)}{p !\left(\gamma+\gamma^{\prime}-1 ; p\right)} x^{p} F\left(\beta^{\prime},-p ; 1-\beta-p ; 1\right),
\end{aligned}
$$

and from this formula (1) follows by applying Gauss's theorem. The proof of (2) is the same as the proof of (1).

To prove (3), expand each hypergeometric function on the left hand side of (3) and change the order of integration and summation; then it becomes

$$
\begin{aligned}
\sum_{m=0}^{\infty} \sum_{n=0}^{\infty} m ! n !\left(\frac{1}{2} \alpha+\frac{1}{2} \beta+\frac{1}{2} ; m\right)\left(\frac{1}{2} \alpha^{\prime}+\frac{1}{2} \beta^{\prime}+\frac{1}{2} ; n\right) \\
\quad \times \frac{1}{2 \pi i} \int \Gamma(\alpha+m+s) \Gamma\left(\alpha^{\prime}+n+s\right) \Gamma(\beta+m-s) \Gamma\left(\beta^{\prime}+n-s\right) d s .
\end{aligned}
$$

From (6), it follows that the last integral is equal to

$$
\begin{gathered}
I^{\prime}(\alpha+\beta+2 m) I^{\prime}\left(\alpha+\beta^{\prime}+m+n\right) I^{\prime}\left(\alpha^{\prime}+\beta+m+n\right) \Gamma^{\prime}\left(\alpha^{\prime}+\beta^{\prime}+2 n\right) \\
I^{\prime}\left(\alpha+\alpha^{\prime}+\beta+\beta^{\prime}+2 m+2 n\right)
\end{gathered} .
$$

Thus the left hand side of (3) becomes

$$
\begin{aligned}
& (2 \sqrt{ })^{-1} \Gamma^{\prime}\left(\frac{1}{2} \alpha+\frac{1}{2} \beta+\frac{1}{2}\right) I\left(\frac{1}{2} \alpha^{\prime}+\frac{1}{2} \beta^{\prime}+\frac{1}{2}\right) \\
& \quad \times \sum_{m=0}^{\infty} \sum_{n=0}^{\infty} \frac{\Gamma\left(\frac{1}{2} \alpha+\frac{1}{2} \beta+m\right) \Gamma\left(\frac{1}{2} \alpha^{\prime}+\frac{1}{2} \beta^{\prime}+n\right)}{m ! n ! \Gamma\left(\frac{1}{2} \alpha+\frac{1}{2} \alpha^{\prime}+\frac{1}{2} \beta+\frac{1}{2} \beta^{\prime}+\frac{1}{2}+m+n\right)} \\
& \quad \times \frac{\Gamma\left(\alpha+\beta^{\prime}+m+n\right) \Gamma\left(\alpha^{\prime}+\beta+m+n\right)}{\Gamma\left(\frac{1}{2} \alpha+\frac{1}{2} \alpha^{\prime}+\frac{1}{2} \beta+\frac{1}{2} \beta^{\prime}+m+n\right)} x^{m+n} \\
& =\Gamma\left(\alpha+\beta^{\prime}\right) \Gamma\left(\alpha^{\prime}+\beta\right) \Gamma(\alpha+\beta) \Gamma\left(\alpha^{\prime}+\beta^{\prime}\right)\left\{\Gamma\left(\alpha+\alpha^{\prime}+\beta+\beta^{\prime}\right)\right\}^{-1} \\
& \quad \times \sum_{p=0}^{\infty} p !\left(\frac{1}{2} \alpha+\frac{1}{2} \alpha^{\prime}+\frac{1}{2} \beta+\frac{1}{2} \beta^{\prime} ; p\right)\left(\frac{1}{2} \alpha+\frac{1}{2} \alpha^{\prime}+\frac{1}{2} \beta+\frac{1}{2} \beta^{\prime}+\frac{1}{2} ; p\right) \\
& \quad \times F\left(\frac{1}{2} \alpha+\frac{1}{2} \beta,-p ; 1-\frac{1}{2} \alpha^{\prime}-\frac{1}{2} \beta^{\prime}-p ; 1\right) ;
\end{aligned}
$$

and from this, formula (3) follows by applying Gauss's theorem. From the proof of (3), the following formula can be deduced: 
( 8 )

$$
\begin{aligned}
& \frac{1}{2 \pi i} \int \Gamma(\alpha+s) \Gamma^{\prime}(\beta-s) \Gamma\left(\alpha^{\prime}+s\right) \Gamma\left(\beta^{\prime}-s\right) \\
& \quad \times F\left(\alpha+s, \beta-s ; \frac{1}{2} \alpha+\frac{1}{2} \beta ; x\right) F\left(\alpha^{\prime}+s, \beta^{\prime}-s ; \frac{1}{2} \alpha^{\prime}+\frac{1}{2} \beta^{\prime} ; x\right) d s \\
& =\Gamma(\alpha+\beta) \Gamma\left(\alpha^{\prime}+\beta^{\prime}\right) \Gamma\left(\alpha+\beta^{\prime}\right) \Gamma\left(\alpha^{\prime}+\beta\right)\left\{\Gamma\left(\alpha+\alpha^{\prime}+\beta+\beta^{\prime}\right)\right\}^{-1} \\
& \quad \times{ }_{3} F_{2}\left[\begin{array}{c}
\alpha+\beta^{\prime}, \alpha^{\prime}+\beta, \frac{1}{2}\left(\alpha+\alpha^{\prime}+\beta+\beta^{\prime}+2\right) ; x \\
\frac{1}{2}\left(\alpha+\alpha^{\prime}+\beta+\beta^{\prime}\right), \frac{1}{2}\left(\alpha+\alpha^{\prime}+\beta+\beta^{\prime}+1\right)
\end{array}\right],
\end{aligned}
$$

where $\alpha+\alpha^{\prime}+\beta+\beta^{\prime} \neq 0,-1,-2, \cdots$.

The proof of (4) is the same as the proof of (3), while formula (5) can be deduced by substituting for each hypergeometric function on the left hand side an integral of Barnes's type and changing the order of integration.

Finally, I may mention the following formula which involves a generalized hypergeometric function,

(9) $\frac{1}{2 \pi i} \int \Gamma(\alpha+s) \Gamma(\beta+s) \Gamma\left(\alpha^{\prime}-s\right) \Gamma\left(\beta^{\prime}-s\right)$

$$
\begin{aligned}
& \times F(\alpha+s, \beta+s ; \gamma ; x) F\left(\alpha^{\prime}-s, \beta^{\prime}-s ; \gamma^{\prime} ; x\right) d s \\
= & \Gamma\left(\alpha+\alpha^{\prime}\right) \Gamma\left(\alpha+\beta^{\prime}\right) \Gamma\left(\beta+\alpha^{\prime}\right) \Gamma\left(\beta+\beta^{\prime}\right)\left\{\Gamma\left(\alpha+\alpha^{\prime}+\beta+\beta^{\prime}\right)\right\}^{-1} \\
& \times{ }_{6} F_{5}\left[\begin{array}{c}
\alpha+\alpha^{\prime}, \alpha+\beta^{\prime}, \beta+\alpha^{\prime}, \beta+\beta^{\prime}, \frac{1}{2}\left(\gamma+\gamma^{\prime}-1\right), \frac{1}{2}\left(\gamma+\gamma^{\prime}\right) ; x \\
\gamma, \gamma^{\prime}, \gamma+\gamma^{\prime}-1, \frac{1}{2}\left(\alpha+\alpha^{\prime}+\beta+\beta^{\prime}\right), \frac{1}{2}\left(\alpha+\alpha^{\prime}+\beta+\beta^{\prime}+1\right)
\end{array}\right],
\end{aligned}
$$

where $\gamma+\gamma^{\prime}-1 \neq 0,-1,-2, \cdots, \alpha+\alpha^{\prime}+\beta+\beta^{\prime} \neq 0,-1,-2, \cdots$ and either $\gamma$ or $\gamma^{\prime}$ is not zero or a negative integer.

3. Confluent forms of addition theorems. In (1) take $\beta^{\prime}=\beta$, write $x / \beta$ for $x$ and let $\beta \rightarrow \infty$ to get

$$
\begin{aligned}
& -\frac{1}{2 \pi i} \int_{k-i \infty}^{k+i \infty} \frac{\Gamma(\alpha+s) \Gamma\left(\alpha^{\prime}-s\right)}{\Gamma(\gamma+s) \Gamma\left(\gamma^{\prime}-s\right)} F(\alpha+s ; \gamma+s ; x) F\left(\alpha^{\prime}-s ; \gamma^{\prime}-s ; x\right) d s \\
& \quad=\frac{\Gamma\left(\alpha+\alpha^{\prime}\right) \Gamma^{\prime}\left(\gamma+\gamma^{\prime}-\alpha-\alpha^{\prime}-1\right)}{\Gamma\left(\gamma+\gamma^{\prime}-1\right) \Gamma(\gamma-\alpha) \Gamma\left(\gamma^{\prime}-\alpha^{\prime}\right)} F\left(\alpha+\alpha^{\prime} ; \gamma+\gamma^{\prime}-1 ; 2 x\right),
\end{aligned}
$$

where $\mathfrak{L}(k+\alpha)>0, \mathfrak{2}(k+\gamma)>0, \mathfrak{2}\left(\alpha^{\prime}-k\right)>0, \mathfrak{2}\left(\gamma^{\prime}-k\right)>0, \gamma+\gamma^{\prime}-1 \neq 0,-1$, $-2, \cdots ; \gamma-\alpha \neq 0,-1,-2, \cdots$ and $\gamma^{\prime}-\alpha^{\prime} \neq 0,-1,-2, \cdots$.

In (2), take $\gamma^{\prime}=\gamma$, write $x / \gamma$ for $x$ and let $\gamma \rightarrow \infty$, to get

$$
\frac{1}{2 \pi i} \int_{k-i \infty}^{k+i \infty} \frac{\Gamma(\alpha+s) \Gamma\left(\alpha^{\prime}-s\right)}{\Gamma(\beta+s) \Gamma\left(\beta^{\prime}-s\right)} F(\beta-\alpha ; \beta+s ; x) F\left(\beta^{\prime}-\alpha^{\prime} ; \beta^{\prime}-s ; x\right) d s
$$




$$
=\frac{\Gamma\left(\alpha+\alpha^{\prime}\right) I^{\prime}\left(\beta+\beta^{\prime}-\alpha-\alpha^{\prime}-1\right)}{I^{\prime}(\beta-\alpha) \Gamma^{\prime}\left(\beta^{\prime}-\alpha^{\prime}\right) \Gamma\left(\beta+\beta^{\prime}-1\right)} F\left(\beta+\beta^{\prime}-\alpha-\alpha^{\prime}-1 ; \beta+\beta^{\prime}-1 ; 2 x\right),
$$

where $\Re(k+\alpha)>0, \mathfrak{H}(k+\beta)>0, \mathfrak{N}\left(\alpha^{\prime}-k\right)>0, \Re\left(\beta^{\prime}-k\right)>0, \quad \beta-\alpha \neq 0,-1$, $-2, \cdots ; \beta^{\prime}-\alpha^{\prime} \neq 0,-1,-2, \cdots$ and $\beta+\beta-1 \neq 0,-1,-2, \cdots$.

Finally in (4), take $\beta^{\prime}=\beta$; write $x / \beta$ for $x$ and let $\beta \rightarrow \infty$, to get

$$
\begin{aligned}
\frac{1}{2 \pi i} \int_{k-i \infty}^{\kappa+i \infty} \Gamma(\gamma+s) \Gamma\left(\gamma^{\prime}-s\right) \Gamma^{\prime}(\alpha+s) \Gamma\left(\alpha^{\prime}-s\right) \\
\quad \times F\left(\alpha+s ; \alpha+\gamma^{\prime} ; x\right) F\left(\alpha^{\prime}-s ; \alpha^{\prime}+\gamma ; x\right) d s \\
=\Gamma\left(\gamma+\gamma^{\prime}\right) \Gamma\left(\alpha+\alpha^{\prime}\right) \Gamma\left(\alpha+\gamma^{\prime}\right) \Gamma\left(\alpha^{\prime}+\gamma\right)\left\{\Gamma\left(\alpha+\alpha^{\prime}+\gamma+\gamma^{\prime}\right)\right\}^{-1} \\
\quad \times F\left(\alpha+\alpha^{\prime} ; \alpha+\alpha^{\prime}+\gamma+\gamma^{\prime} ; 2 x\right),
\end{aligned}
$$

where $\Re(k+\gamma)>0, \Re(\alpha+k)>0, \Re\left(\gamma^{\prime}-k\right)>0, \Re\left(\alpha^{\prime}-k\right)>0$, and $\alpha+\alpha^{\prime}+\gamma+\gamma^{\prime}$ $\neq 0,-1,-2, \cdots$. 



\section{PACIFIC JOURNAL OF MATHEMATICS}

\section{EDITORS}

David Gilbarg

Stanford University

Stanford, California

R. A. Beaumont

University of Washington

Seattle 5 , Washington
A. L. Whiteman

University of Southern California Los Angeles 7, California

E. G. Straus

University of California

Los Angeles 24, California

\section{ASSOCIATE EDITORS}
E. F. BECKENBACH
C. E. BURGESS
M. HALL
E. HEWITT
A. HORN
V. GANAPATHY IYER
R. D. JAMES
M. S. KNEBELMAN

L. NACHBIN

I. NIVEN

T. G. OSTROM

H. L. ROYDEN
M. M. SCHIFFEI

G. SZEKERES

F. WOLF

K. YOSIDA

\section{SUPPORTING INSTITUTIONS}

UNIVERSITY OF BRITISH COLUMBIA

CALIFORNIA INSTITUTE OF TECHNOLOGY

UNIVERSITY OF CALIFORNIA

MONTANA STATE UNIVERSITY

UNIVERSITY OF NEVADA

OREGON STATE COLLEGE

UNIVERSITY OF OREGON

OSAKA UNIVERSITY

UNIVERSITY OF SOUTHERN CALIFORNIA
STANFORD UNIVERSITY

UNIVERSITY OF TOKYO

UNIVERSITY OF UTAH

WASHINGTON STATE COLLEGE

UNIVERSITY OF WASHINGTON

AMERICAN MATHEMATICAL SOCIETY

CALIFORNIA RESEARCH CORPORATION

HUGHES AIRCRAFT COMPANY

THE RAMO-WOOLDRIDGE CORPORATION

Printed in Japan by Kokusai Bunken Insatsusha

(International Academic Printing Co., Ltd.), Tokyo, Japan 


\section{Pacific Journal of Mathematics}

\section{Vol. 8, No. 1 \\ March, 1958}

Shimshon A. Amitsur, Commutative linear differential operators ......... 1

Masahiko Atsuji, Uniform continuity of continuous functions of metric

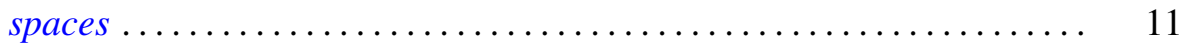

S. P. Avann, A numerical condition for modularity of a lattice . . . . . . . . . 17

Raymond G. D. Ayoub, A mean value theorem for quadratic fields........ 23

Errett Albert Bishop, Subalgebras of functions on a Riemann surface ..... . 29

Shaul Foguel, The relations between a spectral operator and its scalar

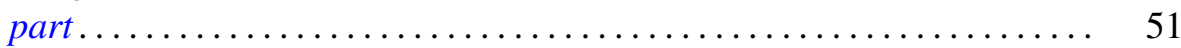

John Rolfe Isbell, Euclidean and weak uniformities ................. 67

Samuel Karlin and James L. McGregor, Many server queueing processes with Poisson input and exponential service times .............. 87

Paul Joseph Kelly and Ernst Gabor Straus, Curvature in Hilbert geometries....................................... 119

John W. Lamperti, Stationary measures for certain stochastic processes . . . 127

Richard Scott Pierce, Distributivity and the normal completion of Boolean

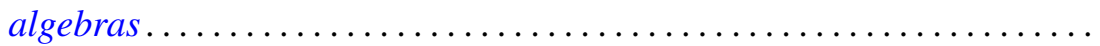

F. M. Ragab, Transcendental addition theorems for the hypergeometric

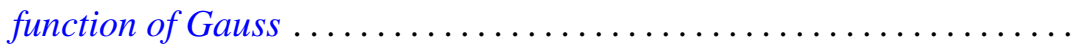

William T. Reid, Principal solutions of non-oscillatory self-adjoint linear differential systems ................................ 147

Maurice Sion, On general minimax theorems .................... 171

Chien Wenjen, On semi-normed ${ }^{*}$-algebras .................... 177 